Review

\title{
New Transfection Agents Based on Liposomes Containing Biosurfactant MEL-A
}

\author{
Mamoru Nakanishi *, Yoshikazu Inoh and Tadahide Furuno \\ School of Pharmacy, Aichi Gakuin University, 1-100 Kusumoto-cho, Chikusa-ku, Nagoya 464-8650, \\ Japan; E-Mails: inoh@dpc.agu.ac.jp (Y.I.); furuno@dpc.agu.ac.jp (T.F.) \\ * Author to whom correspondence should be addressed; E-Mail: mamoru@dpc.agu.ac.jp; \\ Tel.: +81-52-757-6772; Fax: +81-52-757-6799.
}

Received: 27 June 2013; in revised form: 29 July 2013 / Accepted: 8 August 2013 /

Published: 16 August 2013

\begin{abstract}
Nano vectors are useful tools to deliver foreign DNAs, oligonucleotides, and small interfering double-stranded RNAs (siRNAs) into mammalian cells with gene transfection and gene regulation. In such experiments we have found the liposomes with a biosurfacant mannosylerythriol lipid (MEL-A) are useful because of their high transfer efficiency, and their unique mechanism to transfer genes to target cells with the lowest toxicity. In the present review we will describe our current work, which may contribute to the great advance of gene transfer to target cells and gene regulations. For more than two decades, the liposome technologies have changed dramatically and various methods have been proposed in the fields of biochemistry, cell biology, biotechnology, and so on. In addition, they were towards to pharmaceutics and clinical applications. The liposome technologies were expected to use gene therapy, however, they have not reached a requested goal as of yet. In the present paper we would like to present an approach using a biosurfactant, MEL-A, which is a surface-active compound produced by microorganisms growing on water-insoluble substrates and increases efficiency in gene transfection. The present work shows new transfection agents based on liposomes containing biosurfactant MEL-A.
\end{abstract}

Keywords: biosurfactant; cationic liposome; membrane fusion; siRNA; CLSM 


\section{Introduction}

Gene transfection and gene regulation refers to the transmission of DNAs or small (21-23 nucleic acid) interfering double-stranded RNAs (siRNAs) to regulate specific gene expression in the target cells or organs and are very important in biosciences, pharmaceutics, and clinical applications. Over the past two decades, various non-viral vectors have been examined to avoid "Tragic setback" with virus vectors, such as adenoviruses and retroviruses [1]. However, the success of gene transfection and gene regulation by non-viral vectors did not proceed well because of their low transfection efficiency [2-8].

Ten years ago, we reported a short review article for the non-viral liposome vectors made of cationic cholesterol derivatives [9]. Cationic cholesterol derivatives are composed of three distinct parts, a cholesteryl skeleton, a spacer arm between cationic amino residue and cholesterol, and a cationic amino terminal group. The derivatives with different combinations of these three parts were also reported and some of them have rather high transfection efficiency [4-9]. There, we found that the liposomes made of a cholesterol derivative with a hydroxyethyl amino head group are much more effective than those of the liposomes with the conventional cationic cholesterol derivative of alkylamino head groups as shown in Figure 1. It seemed that the liposome vectors must preserve the balance of molecular interaction between the hydrophilicity and hydrophobicity to transfer genes to the target cells [5]. Therefore, we studied the molecular mechanism of gene transfection with molecular imaging and that we found several accessory molecules with the liposomes were able to promote the transfer of DNAs into the target cells [9].

Figure 1. Structures of cholesteryl-3 $\beta$-carboxyamidoethylene- $N$-hydroxyethylamine, $\mathrm{OH}-\mathrm{Chol}$ and commercially available cationic cholesterol DC-Chol (lower).

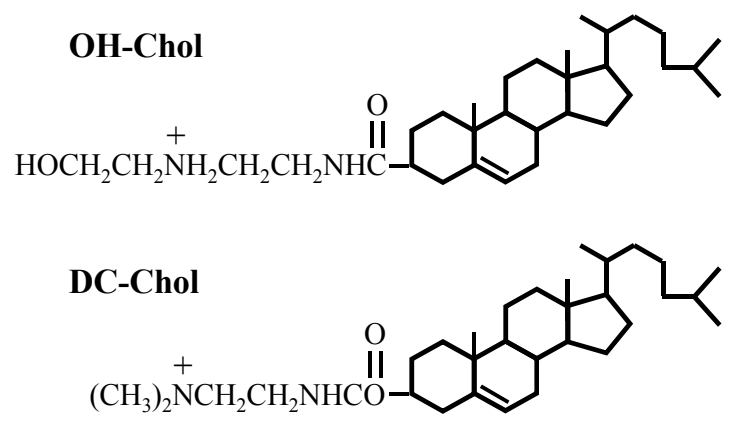

A biosurfactant, mannosylerythriol lipid A (MEL-A), is a major component of MELs produced by yeast strain Candida antarctica T-34 [10,11]. Biosurfactants have received great attention due to their unique properties such as low toxicity, biodegradability, biological activities, and self-assembling properties [12-14]. We found that MEL-A dramatically increased the efficiency of gene transfection, in 2001 [15], and clarified why the liposomes with MEL-A were able to increase the efficiency of the gene transfection [16]. The great transfection efficiency was due to the membrane fusion between liposomes and the target cells similar to some RNA virus vectors [16-19]. That is, the liposomes with MEL-A introduce foreign genes into the target cells by two different pathways; one is a pathway with the endocytosis of the vector/gene complexes into the target cells and another is a pathway of the membrane fusion, rapidly and safely.

In addition, RNA interference (RNAi) is mediated by siRNAs that originate from longer double-stranded DNAs (shRNAs) of endogenous or exogenous origin after cleavage by Dicer [20]. 
These siRNA molecules are then incorporated into a siRNA-induced silencing complex (RISC) where the shRNAs are cleaved to release single-stranded siRNAs [21-23]. RNAi is a promising tool for gene therapy against various diseases because siRNA has high targeting specificity for selective proteins without side effects [24-27]. Furthermore, siRNA is more stable and works at a lower concentration, for a longer time, than single-stranded oligonucleotides like antisense DNAs [28]. Despite these promising properties of siRNA therapeutics, several obstacles remain to be overcome. One of them is the poor cellular uptake of siRNA and accumulation of exogenous siRNA in endosomes [29]. Thus, a more efficient and safer system for siRNAs and shRNAs is needed for these molecules to make a significant clinical impact [30-35].

\section{Liposome Vectors with Biosurfactants MEL-A}

Non-viral liposome vectors were made of the complex of DOPE (dioleoyl phosphatidyl ethanolamine) and a cationic cholesterol derivative, cholesteryl-3 $\beta$-carboxyamidoethylene- $N$-hydroxy ethylamine (OH-Chol) or commercially available cationic cholesterol (DC-Chol) to compare the gene transfection efficiency as also shown in Figure 1 [5,9]. A structure of the biosurfactant MEL-A,

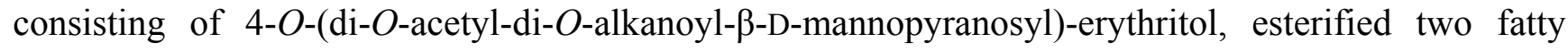
acids and two acetic acids and is shown in Figure 2.

Figure 2. A structure of biosurfactant MEL-A which consists of 4-O-[ $\beta$-D-mannopyranosyl] meso-erythritol], esterified two fatty acids and two acetic acids.

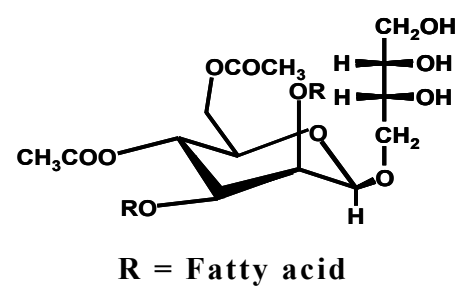

MEL-A-containing liposome vectors with a cholesterol derivative $\mathrm{OH}-\mathrm{Chol}$ or DC-Chol were complexed with plasmid DNAs for luciferase (pGL3) or siRNAs targeting luciferase (siGL3) (sense: 5'-CUUACGCUGAGUACUUCGATT-3' and antisense 5'-UCGAAGUACUCGCGUAAGTT-3'). Effects of transfection (not transfecting) activity were measured by luciferase activity or the silencing effects of target protein to the target cells [22,23]. The MEL-A greatly increased transfection activities in the target cells using both liposome vectors composed of $\mathrm{OH}-\mathrm{Chol}$ and DC-Chol in comparison with the vectors without MEL-A as shown in Figure 3. In addition, the liposome vectors of OH-Chol had greater transfection efficiency than those of the DC-Chol.

In the experiments of siRNA silencing effects, we measured the suppression of Bcl-2 expression in B16/BL6 melanoma cells after delivering siRNA targeting Bcl-2 by MEL-A-containing cationic liposomes. To determine the most effective method of siRNA transfection, we measured cytotoxicity and suppression of luciferase expression in luciferase-expressing B16 cells (B16-luc cells) exposed to siGL3 at a fixed concentration with various ratios of MEL-A-containing cationic liposomes. The details were described in the previous paper [36]. As described above, such kinds of characteristics existed both 
in the case of plasmid DNAs and siRNAs (shRNAs) with liposome vectors containing biosurfactant MEL-As [36].

Figure 3. Effects of the liposome vectors with MEL-A on the transfection efficiency in target cells. NIH-3T3 cells were transfected with luciferase gene (pGL3) using two different cationic cholesterol derivatives, OH-Chol and DC-Chol without MEL-A (black bars) and with MEL-A (white bars). Transfection efficiency was measured by luciferase assay and values were normalized to the amount of total protein. Values are mean \pm S.E. $(n=3)$. $* * p<0.01$ compared with cationic liposomes without MEL-A; ${ }^{\#} p<0.01$ compared with DC-Chol by Students $t$ test, respectively. The details were described in our previous paper [16].

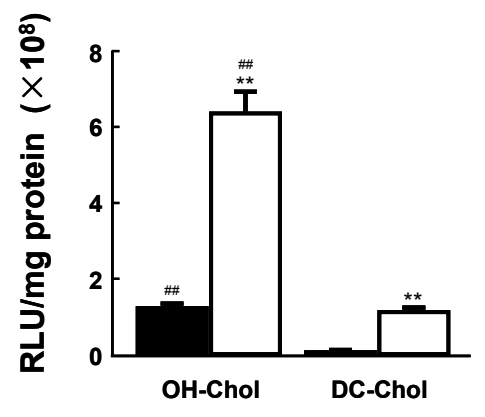

To investigate the cellular uptake mechanism of siRNAs, we studied the subcellular localization of fluorescein-labeled siRNAs after transfection with confocal laser scanning fluorescence microscopy (CLSM). The results revealed that MEL-A-containing cationic liposomes were able to deliver siRNA into the cytoplasm of the target cells very rapidly, and that a short incubation time of 10-30 min was enough to reach the value of the maximum silencing effect, though Lipofectamine ${ }^{\text {TM }}$ RNAiMax (Invitrogen, Carlsbad CA, USA) needed much more time and the silencing effect increased more slowly [36].

\section{Mechanism to Transfer the Liposome/Vector Complexes to Target Cells}

CLSM gave us useful information on how the liposome vectors with MEL-A are very effective to transfer plasmid DNAs and siRNAs into the target cells. Another microscopic method, atomic force microscopy (AFM) is also very useful to understand the molecular mechanism of the gene transfection and the structure of the complex of non-viral vectors and genes. As we described the experimental results by AFM in our previous review article and several papers [9,37,38], we do not describe them in the present paper.

In the present paper we will describe the interesting and unique results of the gene transfection with non-viral vectors with MEL-A through the fluorescence energy transfer and the fluorescence in vitro cell images. Figure 4 shows CLSM images of NIH3T3 cells incubated with fluorescence labeled liposome-DNA complex, using $\mathrm{OH}-\mathrm{Chol}$ as a cholesterol derivative. In the case of liposomes with MEL-A, fluorescence labeled liposomes with MEL-A/antisense DNA complexes were temporarily on the plasma membrane of the target cells, and antisense oligonucleotides were transferred into the nucleus. The distribution of bright fluorescence was observed along the plasma membranes. This suggested that membrane fusion occurred between liposomes and the plasma membrane. To the 
contrary, the fluorescence intensity change was neither observed in the nucleus nor on the plasma membranes at $1 \mathrm{~h}$ incubation with the cationic liposomes without MEL-A [16].

Figure 4. CLSM images of NIH3T3 cells incubated with fluorescence labeled liposome-DNA complex. NIH3T3 cells were incubated with FITC-conjugated oligonucleotides complexed with rhodamine-labeled liposomes composed of OH-Chol without MEL-A (a) and with MEL-A (b) for $1 \mathrm{~h}$. Fluorescence images of liposome (right) and merged images on a transmittance image with pseudo-color (yellow for DNA, blue for liposome, and pink for liposome-DNA-complex) (left) are shown.

(a)
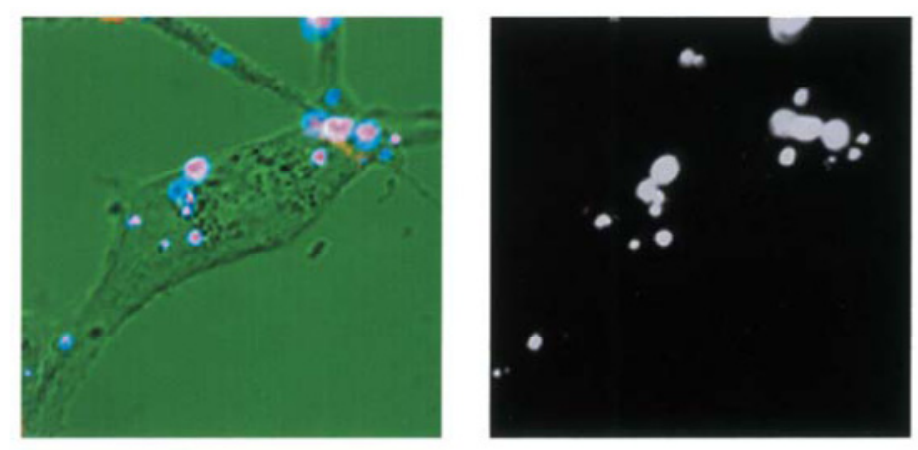

(b)
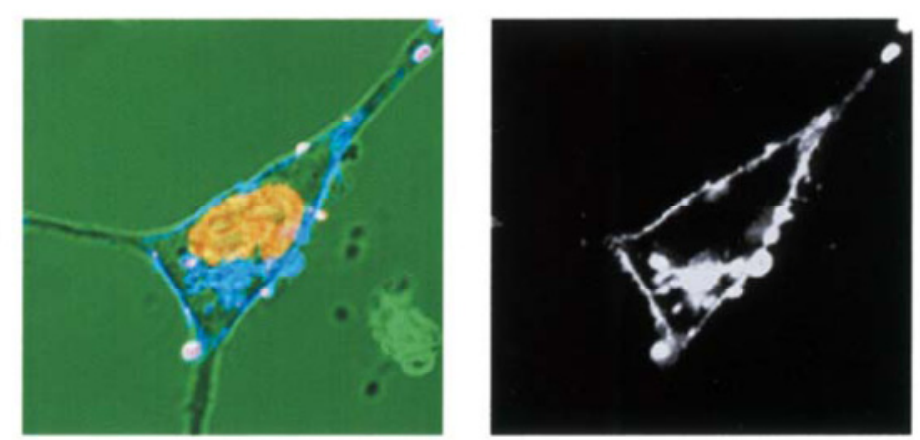

To understand more precisely how membrane fusion occurred between liposome membranes and the target cell membranes, we used two kinds of model membranes to study the effects of biosurfactants on the fluorescence energy transfer. As shown in Figure 5, we studied the effects of MEL-A on the membrane fusion between cationic liposomes and anionic model liposomes by fluorescence spectroscopy. The procedure is shown in Figure 5, schematically [16]. The membrane fusion was measured with the decrease in FRET efficiency between two fluorophores, NBD-PE (donor) and rhodamine-PE (acceptor) in anionic liposomes according to the increase of their distance by mixing with non-fluorescent lipids in cationic liposomes. Therefore, when the membrane fusion occurred between anionic liposomes and cationic liposomes, NBD fluorescence intensity increased at $525 \mathrm{~nm}$ and rhodamine fluorescence at $575 \mathrm{~nm}$ decreased as shown in Figure 5. Figure 5c shows the time course of fluorescence intensity changes of NBD at $525 \mathrm{~nm}$ in anionic liposomes after the addition of cationic liposomes with or without MEL-A. The plateau level of fluorescence intensity after the addition of liposomes with MEL-A (after 10-15 min) increased much more than that in the case without biosurfactants. These results were well consistent with our previous findings that MEL-A-containing cationic liposome membranes were able to facilitate the fusion between the plasma membrane of the target cells and cationic liposomes [16]. 
Figure 5. The decrease in FRET efficiency by membrane fusion from NBD-PE to rhodamine-PE. (a) The schematic representation of decrease in FRET efficiency from NBD-PE to rhodamine-PE by membrane fusion; (b) Fluorescent spectra of NBD and rhodamone in anionic liposomes before (broken line) and at $10 \mathrm{~min}$ after the addition of cationic liposomes containing MEL-A (solid line); (c) The time course of membrane fusion between cationic and anionic liposomes by FRET assay. The cationic liposomes with MEL-A intensity of NBD according to the decrease in FRET efficiency by member fusion were monitored.

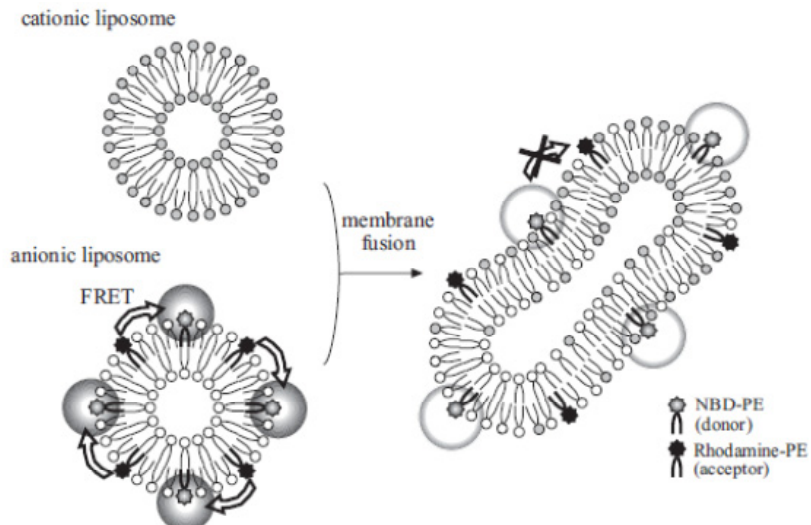

(a)

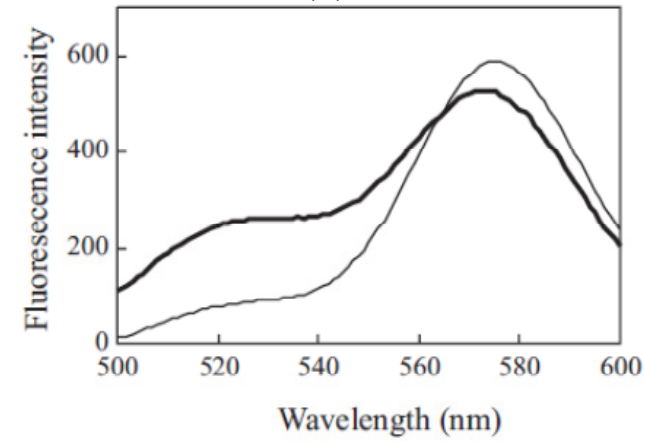

(b)

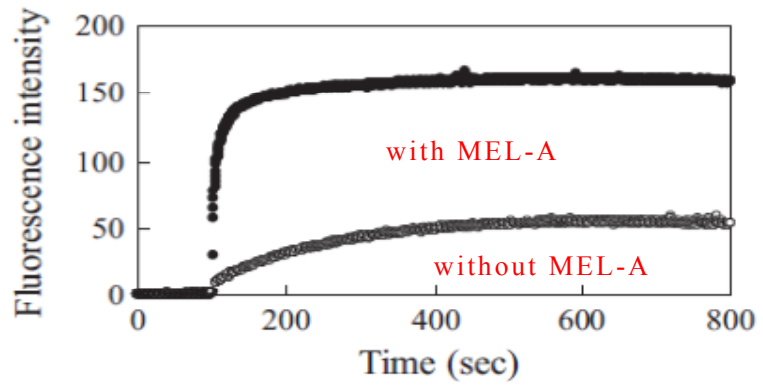

(c)

\section{Membrane Fusion Works for siRNA Transfection}

Figure 6 shows a typical example of CLSM images of B16/BL6 cells treated with MEL-A-containing liposome/siRNAs complexes described above. Here, MEL-A-containing liposome vectors with a cholesterol derivative $\mathrm{OH}-\mathrm{Chol}$ were complexed with siRNAs. Endosomes/lysosomes were stained with LysoTracker Red (red) and siRNAs were stained by fluorescein-conjugated siRNA (green). The details of the experimental procedure were reported in a previous paper [36]. As shown in Figure 6, most of the 
fluorescein-conjugated siRNA fluorescence (green) was separated from endosomes/lysosomes fluorescence (red) when B16/BL6 cells were treated with the MEL-A-containing liposome/siRNAs complexes [36]. The results indicated that MEL-A-containing cationic liposomes were able to deliver siRNAs rapidly into the target cells within 10-30 min of incubation, time shown in Figure 6. It was quite different from the siRNAs that were transfected with Lipofectamine ${ }^{\mathrm{TM}}$ RNAiMax. Here, siRNAs were transferred much slower into the target cells with Lipofectamine ${ }^{\mathrm{TM}}$ RNAiMax, although, after $1 \sim 2 \mathrm{~h}$, both methods reached almost similar levels of silencing effects. This indicated that the cellular uptake mechanism of siRNAs mediated by MEL-A-containing cationic liposomes was quite different from those mediated with Lipofectamine ${ }^{\mathrm{TM}}$ RNAiMax, where the endocytotic pathway was mainly involved in gene transfer to the target cells.

Figure 6. (Upper) Intracellular localization of FAM-labeled siRNA with MEL-A-containing cationic liposomes. Lysotracker Red stained B16/BL6 cells were observed $0.5 \mathrm{~h}$ after incubation with liposome/siRNA. Left is DIC and Right is a CLSM image of FAM-labeled siRNA; (Lower) Time-depended silence effects for the experiments with MEL-A-containing cationic liposomes (blue) and Lipofectamine ${ }^{\mathrm{TM}}$ RNAiMax (red).

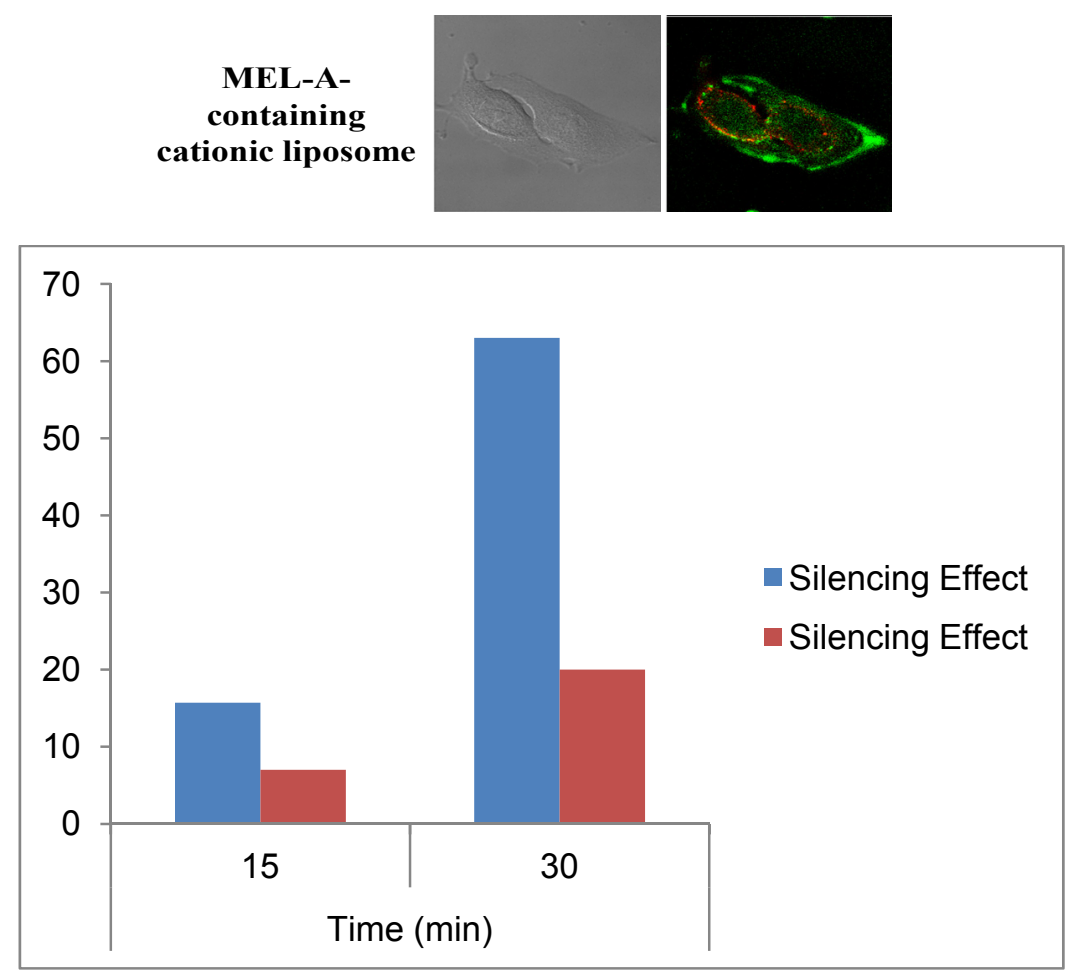

\section{Intracellular Rapid Distribution of Components of Liposome/siRNA Complex}

Finally, we investigate the mechanism of the rapid delivery of siRNAs to the target cells. We studied the individual processes in delivery of siRNAs into the target cells with the liposomes/siRNAs complexes of MEL-A. The typical fluorescence images in siRNAs complexes were transferred into the target cells by the MEL-A-containing cationic liposomes shown in Figure 6. At 10 min after the addition of the liposome-DNA complexes, they attached to the plasma membrane of target NIH-3T3 cells. A small amount of MEL-A was found to start to distribute to the intracellular membranes. 
Rhodamine-conjugated PE, which was a content of the vector, was diffused from the contact site to the plasma membrane of target cells, indicating that the fusion between the liposome and the plasma membrane occurred. Figure $5 \mathrm{c}$ shows representative time-courses of fluorescence intensity changes of NBD-conjugated MEL-A in intracellular membranes, rhodamine-conjugated PE in the plasma membrane, and Cy5-conjugated oligonucleotide in the nucleus. The MEL-A was accumulated in the intracellular membranes continuously after the addition of the complexes, while the fusion of the vectors with the plasma membrane proceeded. After a short period of time, the oligonucleotide DNA was suddenly released from the complexes by the membrane fusion and it was delivered into the nucleus. A schematic model of gene transfer with MEL-A-containing liposome vectors, based on the present experiment, is shown in Figure 5. The membrane fusion between the plasma membranes and the liposome vectors plays a role in gene transfection in the case of liposome vector with MEL-A.

\section{Conclusions}

Biosurfactants are extracellular amphiphilic compounds produced by various kinds of microorganisms and have attracted considerable interest because of their biodegradability, mild productive conditions, and variety of other functions. The numerous advantages of biosurfactants have promoted applications in food, cosmetic, and pharmaceutical industries, and environmental protection and energy-saving technology [10-14]. It is also considered that biosurfactants play important roles as immune-regulators and immune-modulators in adhesion and desorption in cellular systems. In addition to these interesting properties of biosurfactants, we showed that the biosurfactant MEL-A-containing liposome vectors remarkably promoted the efficiency of gene transfection into mammalian cultured cells $[15,16]$.

Further, we showed in the present review that the MEL-A-containing liposome vectors transfected foreign genes into the nucleus of target cells with a shorter incubation time $(10 \sim 30 \mathrm{~m})$ via membrane fusion. The liposome vector has a new pathway of the membrane fusion to transfer genes to the target cells with high rapidity and efficiency, which seems a great technique to avoid the "Tragic setback" with virus vectors [1].

\section{Conflicts of Interest}

The authors declare no conflict of interest.

\section{References}

1. Check, E. Gene therapy: A tragic setback. Nature 2002, 420, 116-118.

2. Felgner, P.L.; Ringold, G.M. Cationic liposome-mediated transfection. Nature 1989, 337, 387-388.

3. Felgner, P.L.; Gadek, T.R.; Holm, M.; Roman, R.; Chan, H.W.; Wenz, M.; Northrop, J.P.; Ringhold, G.M.; Danielson, M. Lipofectin: A highly efficient, lipid-mediated DNA-transfection procedure. Proc. Natl. Acad. Sci. USA 1987, 84, 7413-7417.

4. Gao, X.; Huang, L. A novel cationic liposome reagent for efficient transfection of mammalian cells. Biochem. Biophys. Res. Commun. 1991, 179, 280-285. 
5. Okayama, R.; Noji, M.; Nakanishi, M. Cationic cholesterol with a hydroxyethylamino head group promotes significantly liposome-mediated gene transfection. FEBS Lett. 1997, 408, 232-234.

6. Lee, R.J.; Huang, L. Folate-targeted, anionic liposome-entrapped polylysin-condensed DNA for tumor cell-specific gene transfer. J. Biol. Chem. 1996, 271, 8481-8487.

7. Ledley, F.D. Non-viral gene therapy: The promise of genes as pharamaceutical products. Hum. Gene Ther. 1995, 6, 1129-1144.

8. Farhood, H.; Bottega, R.; Epand, R.M.; Huang, L. Effect of cationic choresterol derivatives on gene transfer and protein kinase C activity. Biochem. Biophys. Acta 1992, 1111, 239-246.

9. Nakanishi, M. New strategy in gene transfection by cationic transfection lipids with a cationic cholesterol. Curr. Med. Chem. 2003, 10, 1289-1296.

10. Desai, J.D.; Banat, I.M. Microbial production of surfactants and their commercial potential. Mol. Biol. Rev. 1997, 61, 47-64.

11. Kitamoto, D.; Akiba, S.; Hioki, T.; Tabuchi, T. Extracellular accumulation of mannnosylerythrytol lipids by a strain of Candida antractica. Agric. Biol. Chem. 1990, 54, 31-36.

12. Kitamoto, D.; Yanagishita, H.; Haraya, K.; Kitamoto, H.K. Contribution of a chain-shortening pathway to the biosynthesis of the fatty acids of mannnosylerythrytol lipid (biosurfactant) in the yeast Cndida Antarctica: Effect of $\beta$-oxidation inhibitors on biosurfactant synthesis. Biotechnol. Lett. 1998, 20, 813-818.

13. Lin, S.C. Biosurfactants: Recent advances. J. Chem. Tech. Biotechnol. 1996, 66, 109-120.

14. Kitamoto, D.; Ghosh, S.; Ourisson, G.; Nakatani, Y. Formation of giant vesicles from diacylmannosylerithritols, and their binding to concanavalin A. Chem. Commun. 2000, 861-862.

15. Inoh, Y.; Kitamoto, D.; Hirashima, N.; Nakanishi, M. Biosurfactants of MEL-A increase gene transfection mediated by cationic liposomes. Biochem. Biophys. Res. Commun. 2001, 289, 57-61.

16. Inoh, Y.; Kitamoto, D.; Hirashima, N.; Nakanishi, M. Biosurfactant MEL-A dramatically increases gene transfection via membrane fusion. J. Control Release 2004, 94, 423-431.

17. Ueno, Y.; Hirashima, N.; Inoh, Y.; Furuno, T.; Nakanishi, M. Characterization of biosurfactant-containing liposomes and their efficiency for gene transfection. Biol. Pharm. Bull. 2007, 30, 169-172.

18. Ueno, Y.; Inoh, Y.; Furuno, T.; Hirashima, N.; Kitamoto, D.; Nakanishi, M. NBD-conjugated biosurfactant (MEL-A) shows a new pathway for transfection. J. Control Release 2007, 123, 247-253.

19. Nakanishi, M.; Inoh, Y.; Kitamoto, D.; Furuo, T. Nano vectors with a biorurfactant for gene transfection and drug delivery. J. Drug. Deliv. Sci. Tech. 2009, 19, 165-169.

20. Elbashir, S.M.; Harborth, J.; Lendeckel, W.; Yalcin, A.; Weber, K.; Tuschl, T. Duplexes of 21-nucleotide RNAs mediate RNA interference in cultured mammalian cells. Nature 2001, 411, 494-498.

21. Sen, G.L.; Blau, H.M. Argonaute 2/RISC resides in sites of mammalian mRNA decay known as cytoplasmic bodies. Nat. Cell Biol. 2005, 7, 633-636.

22. Meister, G.; Landthaler, M.; Patkaniowska, A.; Dorsett, Y.; Teng, G.; Tuschl, T. Human Argonaute2 mediates RNA cleavage targeted by miRNAs and siRNAs. Mol. Cell 2004, 15, 185-197.

23. Meade, B.R.; Dowdy, S.F. Enhancing the cellular uptake of siRNA duplexes following noncovalent packaging with protein transduction domain peptides. Adv. Drug Deliv. Rev. 2008, 60, 530-536. 
24. Jacque, J.M.; Triques, K.; Stevenson, M. Modulation of HIV-1 replication by RNA interference. Nature 2002, 418, 435-438.

25. Zender, L.; Hutker, S.; Liedtke, C.; Tillmann, H.L.; Zender, S.; Mundt, B.; Waltemathe, M.; Gosling, T.; Flemming, P.; Malek, N.P.; et al. Caspase 8 small interfering RNA prevents acute liver failure in mice. Proc. Natl. Acad. Sci. USA 2003, 100, 7797-7802.

26. Poeck, H.; Besch, R.; Maihoefer, C.; Renn, M.; Tormo, D.; Morskaya, S.S.; Kirschnek, S.; Gaffal, E.; Landsberg, J.; Hellmuth, J.; et al. 5'-triphosphate-siRNA: Turning gene silencing and Rig-I activation against melanoma. Nat. Med. 2008, 14, 1256-1263.

27. Akhtar, S.; Benter, I.F. Nonviral delivery of synthetic siRNAs in vivo. J. Clin. Invest. 2007, 17, 3623-3632.

28. Brantl, S. Antisense-RNA regulation and RNA interference. Biochim. Biophys. Acta 2002, 1575, $15-25$.

29. Chiu, Y.L.; Ali, A.; Chu, C.Y.; Cao, H.; Rana, T.M. Visualizing a correlation between siRNA localization, cellular uptake, and RNAi in living cells. Chem. Biol. 2004, 11, 1165-1175.

30. Kogure, K.; Akita, H.; Yamada, Y.; Harashima, H. Multifunctional envelope-type nano device (MEND) as a non-viral gene delivery system. Adv. Drug Deliv. Rev. 2008, 60, 559-571.

31. Han, S.E.; Kang, H.; Shim, G.Y.; Suh, M.S.; Kim, S.J.; Kim, J.S.; Oh, Y.K. Novel cationic cholesterol derivative-based liposomes for serum-enhanced delivery of siRNA. Int. J. Pharm. 2008, $353,260-269$.

32. Negishi, Y.; Endo, Y.; Fukuyama, T.; Suzuki, R.; Takizawa, T.; Omata, D.; Maruyama, K.; Aramaki, Y. Delivery of siRNA into the cytoplasm by liposomal bubbles and ultrasound. J. Control. Release 2008, 132, 124-130.

33. Kim, H.K.; Davaa, E.; Myung, C.S.; Park, J.S. Enhanced siRNA delivery using cationic liposomes with new polyarginine-conjugated PEG-lipid. Int. J. Pharm. 2010, 392, 141-147.

34. Segura, T.; Hubbell, J.A. Synthesis and in vitro characterization of an ABC triblock copolymer for siRNA delivery. Bioconjug. Chem. 2007, 18, 736-745.

35. Ladewig, K.; Nibert, M.; Xu, Z.P.; Gray, P.P.; Lu, G.Q.M. Efficient siRNA delivery to mammalian cells using layered double hydroxide nanoparticles. Biomaterials 2010, 31, 1821-1829.

36. Inoh, Y.; Furuno, T.; Hirashima, N.; Kitamoto, D.; Nakanishi, M. Rapid delivery of small interfering RNA by biosurfactant MEL-A-containing liposomes. Biochem. Biophys. Res. Commun. 2011, 414, 635-640.

37. Kawaura, C.; Furuno, T.; Nakanishi, M. AFM images of cationic liposomes complexed with plasmid. Bioimages 1998, 5, 121-125.

38. Kawaura, C.; Noguchi, A.; Furuno, T.; Nakanishi, M. Atomic force microscopy for studying gene transfection mediated by cationic liposomes with a cationic cholesterol derivative. FEBS Lett. 1998, 421, 69-72.

(C) 2013 by the authors; licensee MDPI, Basel, Switzerland. This article is an open access article distributed under the terms and conditions of the Creative Commons Attribution license (http://creativecommons.org/licenses/by/3.0/). 